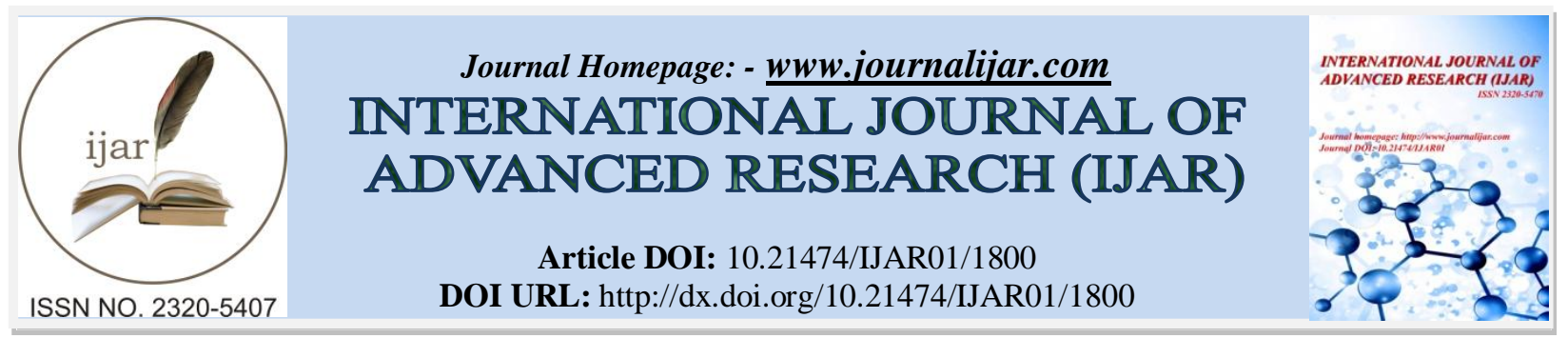

RESEARCH ARTICLE

\title{
COMPARISON OF THE CARIES STATUS USING DMFT, ICDAS II AND CAST INDEX SYSTEM.
}

Dr Ipshita Potlia ${ }^{1}$, Dr P G Naveen Kumar (MDS) ${ }^{2}$, Dr Prashant G M (MDS) ${ }^{3}$, Dr Sushanth V H (MDS) ${ }^{4}$, Dr Mohamed Imranulla (MDS) ${ }^{4}$, Dr Swati Mallick ${ }^{1}$ and Dr Rubel $\mathbf{M}^{1}$.

1. Public Health Dentistry, College of Dental Sciences, Davangere.

2. HOD and Professor, Public Health Dentistry, College of Dental Sciences, Davangere.

3. Professor, Public Health Dentistry, College of Dental Sciences, Davangere.

4. Reader, Public Health Dentistry, College of Dental Sciences Davangere

\section{Manuscript Info}

(..........................

Manuscript History

Received: 12 August 2016

Final Accepted: 22 September 2016

Published: October 2016

Key words:-

Caries status, DMFT, ICDAS II, CAST.

\section{Abstract}

Introduction:-Various methods for caries status evaluation are the Decayed Missing and Filled teeth index (DMFT) and the International Caries Diagnosis \& Assessment System (ICDAS I and II). But serious difficulties in reporting results were encountered while using ICDAS II in epidemiological surveys which necessitated the development of a comprehensive but pragmatic caries assessment index known as CAST INDEX (Caries Assessment Spectrum and Treatment index).

Objectives:-To compare caries status and the amount of time taken for caries status evaluation using DMFT, ICDAS II and CAST index.

Materials and Methods: Two hundred and fifty three dental students were selected randomly and examined. Examination was done by a single calibrated examiner according to principles of DMFT, ICDAS II and CAST index. Evaluation of teeth status was performed in dry and wet situations according to codes of the system.

Results:- When comparing the indices on the amount of time taken, it was noticed that CAST Index took the least time for evaluating a patient followed by DMFT and ICDAS II respectively and this difference was statistically significant $(p=0.001)$. Statistical analysis was performed using descriptive tests, ANOVA, Kruskal Wallis test and data was analyzed using SPSS version 22.

Conclusion:- CAST, the newer index, amongst the three is less time consuming and more useful for investigators in large epidemiological surveys.

Copy Right, IJAR, 2016,. All rights reserved.

\section{Introduction:-}

Dental caries is the most common dental disease known to human race and is recognized by the United Nations as a health burden ${ }^{[1]}$ It is a major cause of tooth loss and pain around the world ${ }^{[2]}$ Hence its detection and assessment is a skill considered as one of the most important competencies required by a dental student. ${ }^{[3]}$

A range of tools for the estimation of dental caries have been presented in the literature. The most widely used is the DMFT index, and other indices used are ICDASI and II and PUFA (pulp, ulcer, fistula and abscess) index etc. 
Although the DMFT index has advantages, they are outweighed by its weaknesses because it does not account for the consequences of untreated cavitated dentine lesions and does not record carious lesions in enamel. Subsequently, the index provides an underestimation of the prevalence and severity of caries. ${ }^{[4]}$ So as to overcome difficulties experienced with the DMFT index and to combine other caries evaluation indices, a new visual and tactile dental caries detection system was developed for international use, designated the International Caries Detection and Assessment System (ICDAS). ${ }^{[5,6]}$ This index was meant to be used in clinical practice and for education, research and epidemiological purposes. ${ }^{[7]}$ However presence of a two digit coding system, use of an air compressor for drying each tooth surface, makes it an expensive and time consuming index difficult for global use in epidemiological surveys. ${ }^{[9]}$ This lead to development of a special index for assessing the very advanced stages of the carious process, known as PUFA index. ${ }^{[9]}$ The pufa PUFA index provides information about the presence of oral conditions consequential from untreated dentine cavities. However it had limited application. ${ }^{[10]}$

In view of the relevant advantages and disadvantages of the DMFT, ICDAS II, and PUFA indices, a new index was proposed to find a reliable, practical, cohesive and easy to read reporting system for presenting results obtained from using both ICDAS II and PUFA indices known as CAST Index. ${ }^{[4]}$

The CAST index describes in a hierarchical way, the complete range or spectrum of carious conditions, from the lack of caries, to the presence of sealant and restoration, lesions in enamel and dentine, lesions penetrating the pulp and tissue surrounding the tooth (abscess/fistulae), and loss of teeth. The hierarchical sequence implies that a high CAST score is considered to represent more severe state than a low CAST score. The index was developed to be used exclusively in epidemiological surveys worldwide. Assessment is performed visually and the use of compressed air is not required. ${ }^{[2]}$

Although studies have been conducted in different countries comparing either two of the DMFT, ICDAS I and II and CAST indices regarding caries score recording, however literature search did not reveal any study comparing the caries status and the time taken to record the same using all of the three indices. Hence there was a need for the study to be performed to understand which of the three index system would take less time and would be more useful for large epidemiological surveys.

\section{Objectives:-}

1) To evaluate and compare the caries status using DMFT, ICDAS II and CAST index.

2) Amount of time taken for evaluation of same using DMFT, ICDAS II and CAST index.

\section{Materials and methods:-}

All the students who were perusing the course in dentistry in College of Dental Sciences, Davangere, fulfilling the inclusions and exclusion criteria were included in the study, which gave a figure of 253 . Informed consent was taken from all the participants before including them in the study. Demographic data were collected in the proforma. All the students who had permanent teeth and gave consent for examination were included in the study. Students undergoing orthodontic treatment and those who were wearing intra-oral appliance were excluded from the study.

\section{Examiner training:-}

A single examiner was trained and calibrated in the DMFT, ICDAS II and CAST Index systems. These sessions lasted for a period of 5 days where the examiner evaluated and scored 20 patients for all the indices. The same set of patients were re-examined at the end of the day to test out intra-examiner reliability which produced a kappa value of 0.84 .

\section{Oral examination:-}

Examinations were performed by a single skilled and calibrated examiner on a dental unit under adequate light conditions using clean dental mirror, probes per the DMFT, ICDAS II and CAST index systems.

All the subjects were examined using DMFT in the first session, ICDAS II in the second session and CAST Index in the third to avoid potential sources of examiner bias. Participants who were absent in this session were examined in the next session. These sessions were held with a time interval of one week between them. 
The current study was approved by the Institutional review board of the dental college. Students were given a consent form explaining the nature of the study and only those who filled in the informed consent were included in the study.

Each examination was initiated from the right maxillary third molar moving anteriorly, passing through left maxillary, left mandibular and finally right mandibular teeth. Each tooth was dried prior to examination. The number of decayed (D), missed (M), and filled (F) teeth were recorded in DMFT form, then the numbers pertaining to $\mathrm{D}, \mathrm{M}$, and $\mathrm{F}$ were added to record the DMFT value.

In ICDAS system, the condition of the teeth were recorded with the sequence as formerly mentioned for DMF system in both moist and dried (using air syringe for 5 seconds) states. As suggested for application of CAST, the tooth surface was not air-dried but, when necessary, excess saliva was removed with cotton rolls or gauzes.

The codes and descriptions that were used for calculating ICDAS II and CAST scores for comparison between the indices are as follows:-

ICDAS codes according to dental conditions: ${ }^{[2]}$

CODE

Code 0

Code 1

Code 2

Code 3

Code 4

Code 5

Code 6

Code 7

Code 8

Code 9
Code Definition

Unfilled and unsealed teeth

Sealant applied but all pits and fissures are not covered

Sealant applied and all pits and fissures are covered

Tooth colored resin or glass ionomer restoration

Amalgam restoration

Stainless steel crown

Porcelain or veneer crown or PFM

Lost or fractured restoration

Temporary restoration

Tooth is missing or has a certain condition
96

97

98

99
The tooth surface is not examinable because of poor accessibility or convenience

The tooth is lost due to caries

The tooth is lost due to reasons other than caries

Unerupted tooth

Caries-Associated with Restorations and Sealants (CARS) Detection Criteria: ${ }^{[2]}$

\section{Code Description}

0

1

2

3

4

5

6
Sound

First visual change in enamel (seen only after prolonged air drying or restricted to within the confines of a pit or fissure)

Distinct visual change in enamel

Localized enamel breakdown (without clinical visual signs of dentin involvement)

Underlying dark shadow from dentin

Distinct cavity with visible dentin

Extensive distinct cavity with visible dentin

The codes and descriptions of the Caries Assessment Spectrum and Treatment (CAST) index: ${ }^{[7]}$

Characteristic Code Description

Sound

Sealed

Restored

Enamel
0

1

2

Code

(1)

3
No visible evidence of a distinct carious lesion is present.

Sealed. Pits and fissures have been at least partially sealed with a sealant material.

A cavity has been restored with an (in)direct restorative material currently without a dentin carious lesion and no fistula/ abscess present Distinct visual change in enamel. A clear carious related discoloration (white or brown in 


$\begin{array}{lll}\text { Dentine } & 4 & \begin{array}{l}\text { color) is visible, including localized enamel } \\ \text { breakdown without clinical visual signs of dentin } \\ \text { involvement. } \\ \text { Internal caries related discoloration in dentine. } \\ \text { The lesion appears as shadows of discolored dentin } \\ \text { visible through enamel which may or may not } \\ \text { exhibit a visible localized breakdown. }\end{array} \\ \text { Distinct cavitation into dentin. No (expected) pulpal } \\ \text { involvement is present. } \\ \text { Involvement of pulp chamber. Distinct cavitation } \\ \text { reaching the pulp chamber or only root fragments are } \\ \text { present } \\ \text { Abscess / Fistula. A pus containing swelling or a pus } \\ \text { releasing sinus tract related to a tooth with pulpal } \\ \text { involvement due to dental caries is present } \\ \text { The tooth has been removed because of dental caries } \\ \text { Does not match with any other categories }\end{array}$

\section{Statistical analysis:-}

Data was entered in Micro-Soft excel 2007 and was analysed using SPSS version 22, $p$ value $\leq 0.05$ was considered statistically significant. The power of the study was fixed at $80 \%$ and confidence interval was set at $95 \%$. Descriptive analysis was done along with Kruskal Walis test to evaluate caries status of the population using the three indices and analysis of variance was used to evaluate the time taken to record the three indices followed by Tukeys post hoc analysis. The scores obtained using the CAST index was converted into DMFT index for easy comparison amongst the indices.

\section{Results:-}

Among the total number of involved people in this study, 153 were females and 100 were males. The mean DMFT for the participants was 2.29. It was observed that $50.66 \%$ of participants had decayed teeth, $45.33 \%$ had filled teeth and only $3.33 \%$ had missing teeth(graph 1 , table 1 ).

While evaluating the results of the ICDAS II systems it was observed that $54 \%$ of the participants had established decay (i.e cavitated lesion) and $84 \%$ of the participants had developed initial caries (graph 2). It was observed that all subjects had at least one non-intact tooth surface i.e none of the participants had a code 00 (sound tooth surfaces) in their dental surfaces. It was also noticed that $64 \%$ of the participants had filled teeth and most commonly used filling was the glass ionomer cement and $6 \%$ had missing teeth (graph 1, table 1).

On looking into the results as obtained by the CAST index it was observed that it gave almost similar results as observed using DMFT index, with decayed, missing and filled teeth being about 50.66\%, 3.33\% and 48\% respectively among the participants (graph 1 , table 1 ).

When the three index systems were compared on the amount of time taken to record them using the analysis of variance (graph 3) a highly significant difference was noticed with $p$ value $<0.001$ (table 2). On performing Tukeys post hoc analysis it was seen that, CAST index took least amount of time, followed by DMFT index which was followed by ICDAS II index system.

Table 1:- Percentage distribution of the decay, missing and filled components using three different indices.

\begin{tabular}{|l|l|l|l|}
\hline INDEX & DECAY $(\%)$ & MISSING (\%) & FILLED (\%) \\
\hline DMFT & 50.66 & 3.33 & 45.33 \\
\hline ICDAS - II & 54 & 6 & 64 \\
\hline CAST & 50.66 & 3.33 & 48 \\
\hline
\end{tabular}

*Statistical analysis by Kruskal Wallis showed no significant difference between the groups $p=0.368$ 
Graph 1:- Comparison of the decay, missing and filled components evaluated using three different indices.

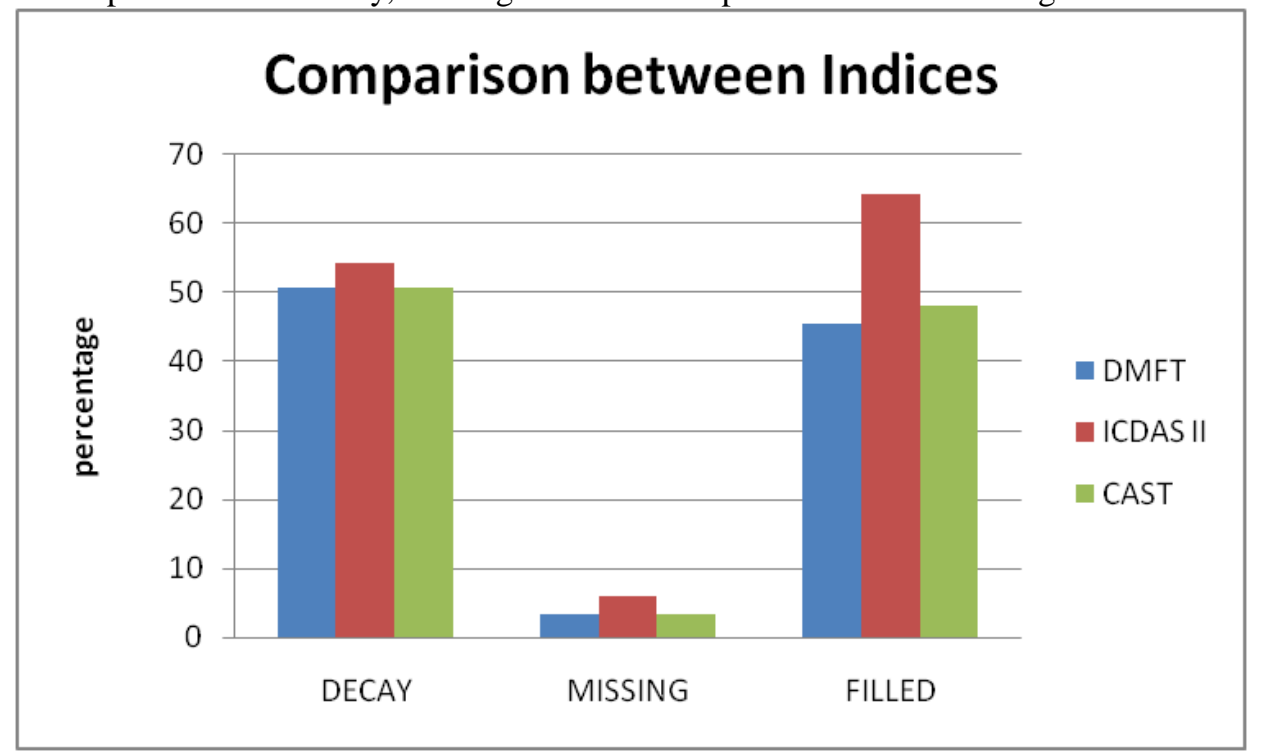

Graph 2:- Distribution of initial and established decay using ICDAS II.

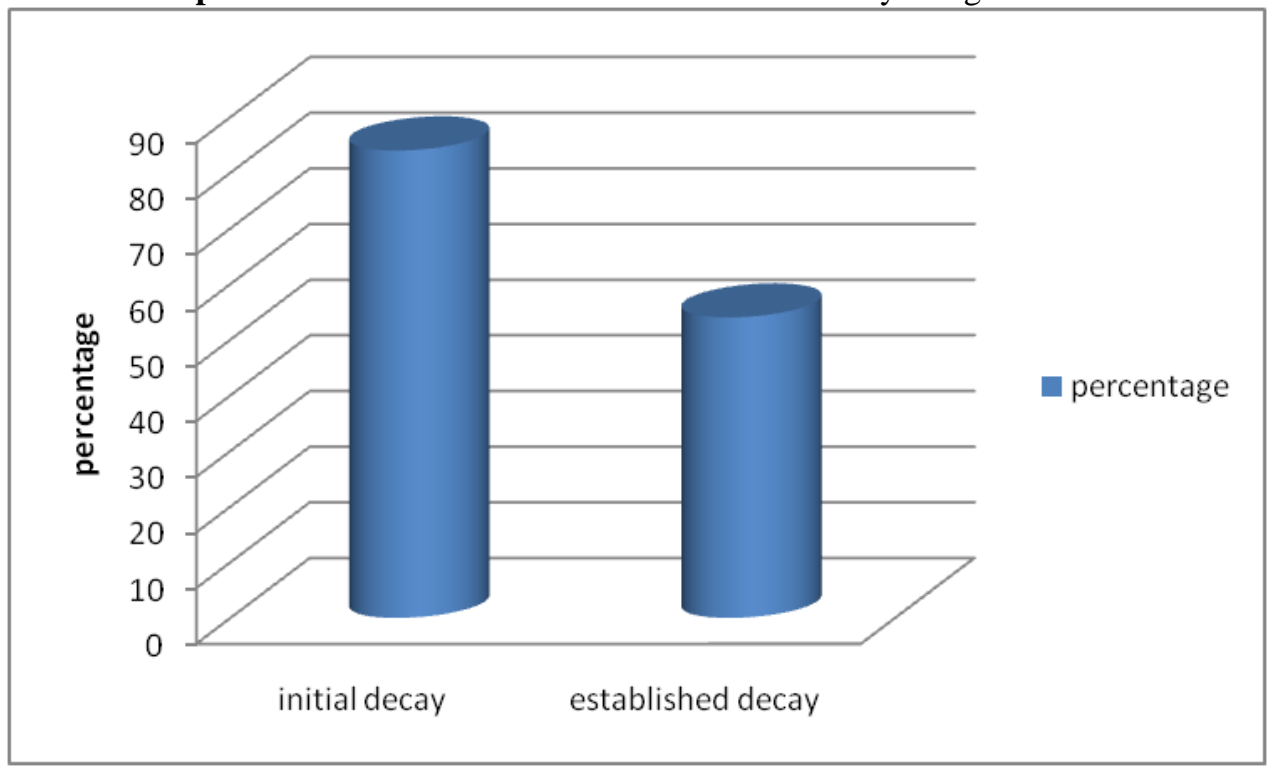

Table 2:- Mean time taken to evaluate and record the 3 indices.

\begin{tabular}{|l|l|l|}
\hline INDEX GROUP & \multirow{3}{*}{$\begin{array}{l}\text { TIME TAKEN } \\
\text { (in seconds) }\end{array}$} & $\begin{array}{l} \\
3\end{array}<.001$ (H.S) \\
\cline { 1 - 2 } DMFT (1) & $145.46 \pm 17.60$ & \\
\hline ICDAS II (2) & $265.88 \pm 21.43$ & \\
\hline CAST (3) & $69.78 \pm 11.69$ & \\
\hline
\end{tabular}

*Statistical analyses by ANOVA followed by tukeys post hoc analysis, $\mathrm{HS}=$ highly significant 
Graph 3:- Mean time taken to record the three index systems in seconds.

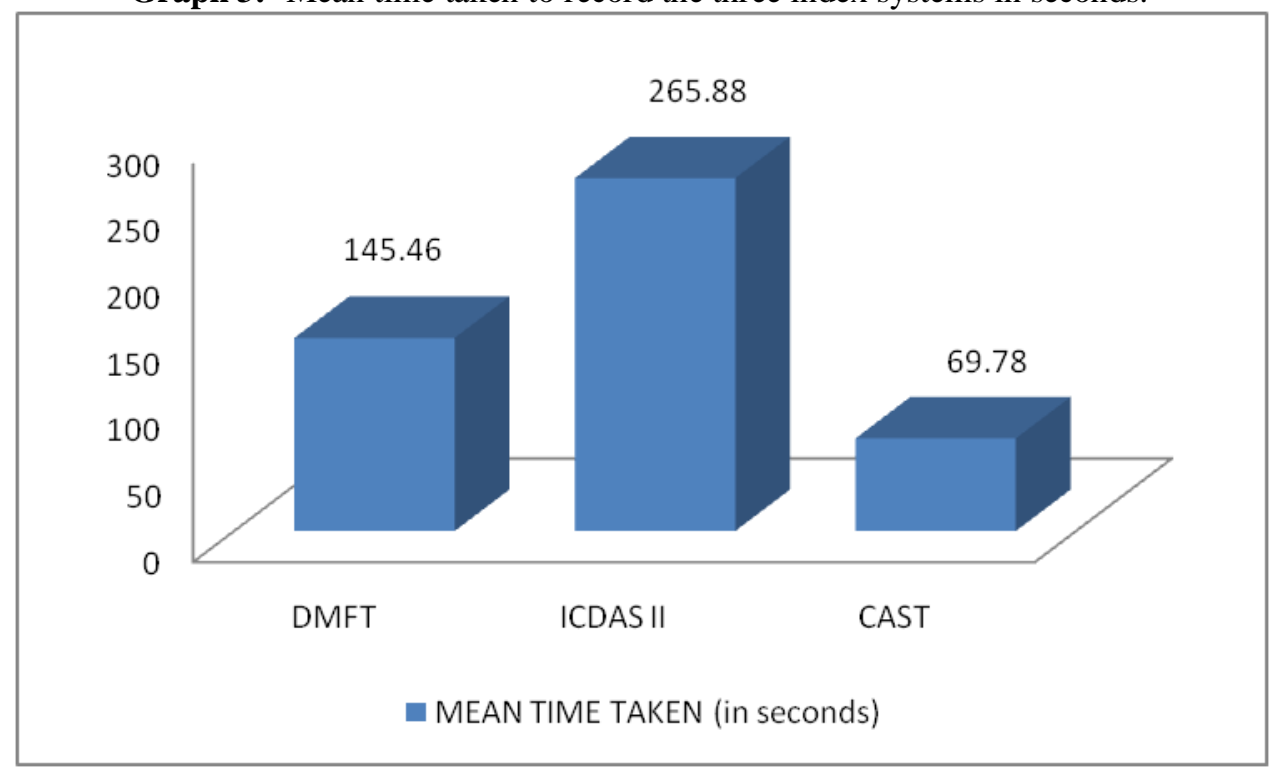

\section{Discussion:-}

Detection of dental caries is an important step in treating this disease ${ }^{[12]}$ Epidemiologically, the most common method for assessment of caries frequency and its state in the DMF index. ${ }^{[13,14]}$ It has the advantage of recording an independent score for every individual, however this number from this evaluation does not give any information about the caries state, stage, depth of penetration, restoration types, and their conditions. In other words it does not inform us regarding the part of the patient care cycle that has been or has to be taken care off.

Hence ICDAS II was developed because the 'old' DMF index was considered obsolete, as early enamel lesions were not part of the DMF index. It was proposed as an alternative to the DMF index.

In this study on using the ICDAS II index it was seen that the decayed, missing and filled components had higher values on the same set of population when compared to the DMFT and CAST index systems (graph 1, table 1). This could be because the ICDAS II index evaluates active as well as inactive carious lesion in contrast to the DMFT and CAST indices which only score for active carious lesions, hence a higher percentage of decayed component is seen on using the index. ${ }^{[7]}$

In similar manner ICDAS II index also evaluates teeth lost for reasons other than caries (orthodontic extractions, etc) which thereby gives a higher percentage for the missing component when compared to the other two index systems (DMFT, CAST) which only evaluate teeth lost due to dental caries (as mentioned in the ICDAS guideline).

The higher percentage for filled teeth in the participants seen on evaluating them using ICDAS II index is because this index also scores the restorations done for reasons other than dental caries, for example restorations done for fractured teeth, midline diastema closure, laminates etc.

However it was difficult to convert the two digit scores obtained on using the ICDAS II index into their corresponding DMFT score and literature search revealed that researchers have encountered problems in reporting the data, necessitating conversion of ICDAS II codes into DMF components, ${ }^{[6,16]}$ or, as an alternative, to produce a table with a long list of possible combinations of the two digits of the system. ${ }^{[6]}$ Therefore, even though ICDAS II index evaluates the patients' caries state, dental conditions and treatment planning with a high degree of reliability, presence of compressed air along with the 2 digit recording system not only made it difficult to analyze statistically, making comparisons with previously done studies using DMF index difficult but it also made it a costly and time consuming index especially for epidemiological studies. Similar problems were noticed in a study conducted by $D e$ Amorim $R G$ et all in brazil, ${ }^{[17]}$ where difficulty in analyzing and converting ICDAS II index into DMF index were noticed. 
The collection of cumulative caries indices available for epidemiological researchers does not cover the total spectrum of carious lesion progression. For example, the ICDAS II system does not cover those carious lesions that involves the pulp and beyond the pulp i.e., the peri-apical region. The PUFA index covers the carious lesions involving the pulp and peri-apical tissues but not the initial caries attack to enamel and dentine. ${ }^{[15]}$

In contrast, the CAST Index integrates the various stages of carious lesion progression and abscesses/fistulae as well as preventive and restorative care in a single-digit coding system. ${ }^{[15]}$ It also gives the advantage of deriving the DMFT score out of it and hence making statistical analysis and comparison with previously done studies using DMFT index much easier.

In this study it was found that the percentage of the participants with decayed, missing and filled teeth had almost similar values when they were evaluated using the DMFT $(50.66 \%, 3.33 \%$ 45.33\% respectively) index and CAST $(50.66 \%, 3.33 \%$ and $48 \%$ respectively) index, making the results obtained more easily comparable.

On the other hand when the same parameters were evaluated on the same set of population using the ICDAS II index, it was seen that the percentage of participants with established caries was $54 \%$ which is slightly more than those seen by the other two indices and it was noteworthy to see that about $84 \%$ of the participants had developed initial caries and so all the participants had at-least one non-intact tooth surface. This shows how the index leads to overestimation of caries, (as the initial carious activity can revert back to normal). When the decayed, missing and filled values from the three indices were subjected to statistical analysis using kruskal wallis no significant difference was noted amongst the three groups (table 1).

The mean time taken to evaluate a participant using the DMFT, ICDAS II and CAST index was approximately $145.46 \pm 17.60,265.88 \pm 21.43,69.78 \pm 11.69$ seconds respectively (graph 3, table 2 ). (It should be noted here that, initially it took more time to calculate CAST index than DMFT index, on undergoing proper training sessions the amount of time taken to calculate CAST index drastically reduced, making it a valuable time saving tool in epidemiological studies.

This was a statistically significant finding, which makes it clear that once properly trained with the index, the time taken to evaluate a participant using CAST index would take even lesser time than DMFT index, which makes it a less time consuming and cost effective tool for large epidemiological surveys/ studies.

Due to the limited number of studies done in the past were comparison was made regarding the time taken to evaluate participants using different index systems, the result of this study could not be compared with previous studies. However it does give us new insight regarding the novel index CAST, it advantages.

\section{Conclusion:-}

The CAST index covers the complete spectrum of the carious process and describes them in a hierarchy of severity (separate codes for sound, sealed, decay in enamel, dentin and pulp).The index is straightforward, easy to apply, least time taking and enables retention of the existing DMF scores. The CAST index for use in epidemiological surveys is very promising as it is less time consuming and more cost effective.

\section{References:-}

1. S. Banava, M. Fattah,MJ. Kharrazifard, T. Safaie, SH. Askarzadeh, M. Safaie Yazdi, B. T. Amaechi, M. Fazlyab. Clinical comparison of dental caries by DMFT and ICDA systems. Journal of Islamic Dental Association of IRAN (JIDAI) /Summer 2012:24(2).

2. Mehta Abhishek. Comprehensive review of caries assessment systems developed over the last decade. RSBO 2012; Jul-Sep:9(3): 316-321.

3. Khalifa S. Al-Khalifa. Use of the International Caries Detection and Assessment System by dental students at the University of Dammam, Saudi Arabia. The Saudi Journal for Dental Research (2015).

4. Burt BA, Baelum V, Fejerskov O. The epidemiology of dental caries. In: Fejerskov O, Kidd E, editors. Dental Caries - The Disease and its Clinical Management, 2nd ed. Frederiksberg: Blackwell Munksgaard; 2008. p. $123-146$. 
5. Pitts N. ICDAS - an international system for caries detection and assessment being developed to facilitate caries epidemiology, research and appropriate clinical management. Community Dent Health 2004 21: $193-$ 198.

6. Ismail AI, Sohn W, Tellez M et al. The International Caries Detection and Assessment System (ICDAS): an integrated system for measuring dental caries. Community Dent Oral Epidemiol 2007 35: 170-178.

7. De Souza AL, van der Sanden WJ, Leal SC, Frencken JE. The Caries Assessment Spectrum and Treatment (CAST) index: face and content validation. Int Dent J.2012 Oct;62(5):270-6.

8. Monse B, Heirich-Weltzien R, Benzian $\mathrm{H}$ et al. PUFA - an index of clinical consequences of untreated dental caries. Community Dent Oral Epidemiol 2010 38: 77-82.

9. Figueiredo MJ, de Amorim RG, Leal SC et al. Prevalence and severity of clinical consequences of untreated dentine carious lesions in children from a deprived area of Brazil. Caries Res 2011 45: 435-442.

10. Oral health survey and fluoride mapping of Bengaluru district 2011.Indian association of public health dentistry, Bengaluru chapter.

11. United Nations General Assembly. Political declaration of thehigh-level meeting of the General Assembly on the Prevention andControl of Non-communicable Diseases. 66th Session. 2011.

12. Diniz MB, Rodrigues JA, Hug I, Cordeiro RC, Lussi A. Reproducibility and accuracy of the ICDAS-II for occlusal caries detection. Com Dent Oral Epidemiol. 2009 Oct; 37(5):399-404.

13. Roberson TM, Heymann H, Edward J. Swift. Sturdevant's art and science of operative dentistry. 4th ed. London; John schrefer; 2006, 108- 112.

14. Fejerskov O, Kidd E. Dental caries: The disease and it's clinical management. 2nd ed. [S.L]: Wiley-Blackwell; 2008, 104,116,118-122,130- 137,142,145.

15. Sushil Phansopkar, Sahana Hegde-Shetiya, Arishka Devadiga, Deepti Agrawal, Amit Mahuli, Simpy MittalMahuli. Face and Content Validation of Caries Assessment Spectrum and Treatment Index among Few Subject Matter Experts in India. International Journal of Dental Health Concerns Vol 1 Issue 1 Jan-Apr 2015.

16. Agustsdottir H, Gudmundsdottir H, Eggertsson H, Jonsson SH, Gudlaugsson JO, Saemundsson SR, Eliasson ST, Arnadottir IB, Holbrook WP (2010) Caries prevalence of permanent teeth: a national survey of children in Iceland using ICDAS. Community Dent Oral Epidemiol 38:299-309.

17. De Amorim RG, Figueiredo MJ, Leal SC, Mulder J, Frencken JE. Caries experience in a child population in a deprived area of Brazil, using ICDAS II. Clin Oral Investig. 2012 Apr;16(2):513-20. 